\title{
Some explicit expressions for the structure coefficients of the center of the symmetric group algebra involving cycles of length three
}

Research Article

\section{Omar Tout}

\begin{abstract}
We use the combinatorial way to give an explicit expression for the product of the class of cycles of length three with an arbitrary class of cycles. In addition, an explicit formula for the coefficient of an arbitrary class in the expansion of the product of an arbitrary class by the class of cycles of length three is given.
\end{abstract}

2010 MSC: 05E15, 20C08

Keywords: The center of the symmetric group algebra, Structure coefficients, Product of conjugacy classes of the symmetric group, Representation theory of the symmetric group

\section{Introduction}

If $n$ is a positive integer, we denote by $\mathcal{S}_{n}$ the symmetric group of permutations on the set $[n]:=$ $\{1,2, \cdots, n\}$. The center of the symmetric group algebra, denoted by $Z\left(\mathbb{C}\left[\mathcal{S}_{n}\right]\right)$, is linearly generated by elements $\mathcal{C}_{\lambda}$, indexed by partitions of $n$, where $\mathcal{C}_{\lambda}$ is the sum of permutations of $[n]$ with cycle-type $\lambda$. The structure coefficients $c_{\lambda \delta}^{\rho}$ describe the product in this algebra, they are defined by the equation:

$$
\mathcal{C}_{\lambda} \mathcal{C}_{\delta}=\sum_{\rho \vdash n} c_{\lambda \delta}^{\rho} \mathcal{C}_{\rho}
$$

In other words, $c_{\lambda \delta}^{\rho}$ counts the number of pairs of permutations $(x, y)$ with cycle-type $\lambda$ and $\delta$ such that $x \cdot y=z$ for a fixed permutation $z$ with cycle-type $\rho$. There is no general formula to compute the coefficients $c_{\lambda \delta}^{\rho}$.

Omar Tout; Department of Mathematics, Faculty of Sciences III, Lebanese University, Tripoli, Lebanon (email: omar-tout@outlook.fr). 
The main two tools to compute the structure coefficients of the center of the symmetric group algebra are the combinatorial way and the character theory. The first one is about studying the cycletype of the product of two permutations which can be a difficult task, see for example [1], [2], [3] and [9]. However, this way had led to a complete formula for the structure coefficients when $\delta$ is the partition coding transpositions, see [8]. By using the representation theory of the symmetric group, the structure coefficients can be expressed in terms of irreducible characters of the symmetric group due to the Frobenius theorem, see [7, Lemma 3.3]. This theorem was used by Goupil and Schaeffer to obtain an explicit formula for the structure coefficients $c_{\lambda \delta}^{\rho}$ when one of the partitions codes the cycles of length $n$, see [6].

In 1958, Farahat and Higman proved in [5, Theorem 2.2] that the coefficients $c_{\lambda \delta}^{\rho}$ are polynomials in $n$ when $\lambda, \delta$ and $\rho$ are fixed partitions, completed with parts equal to 1 to get partitions of $n$. In 1975, Cori showed in his thesis [4] that these coefficients count the number of embeddings of certain graphs into orientable surfaces.

The goal of this paper is to give new explicit formulas for the structure coefficients of the center of the symmetric group algebra using the combinatorial method. We will be interested in computing the product of an arbitrary class of the symmetric group by another coding cycles, especially of lengh three. We hope that these results will be useful to obtain an explicit expression for the product of the class of cycles of length three with an arbitrary class of the symmetric group.

\section{Definitions and the combinatorial way to compute the struc- ture coefficients}

A partition $\lambda$ is a list of integers $\left(\lambda_{1}, \ldots, \lambda_{l}\right)$ where $\lambda_{1} \geq \lambda_{2} \geq \ldots \lambda_{l} \geq 1$. The $\lambda_{i}$ are called the parts of $\lambda$; the size of $\lambda$, denoted by $|\lambda|$, is the sum of all of its parts. If $|\lambda|=n$, we say that $\lambda$ is a partition of $n$ and we write $\lambda \vdash n$. The number of parts of $\lambda$ is denoted by $l(\lambda)$.

We will especially use the exponential notation $\lambda=\left(1^{m_{1}(\lambda)}, 2^{m_{2}(\lambda)}, 3^{m_{3}(\lambda)}, \ldots\right)$, where $m_{i}(\lambda)$ is the number of parts equal to $i$ in the partition $\lambda$. In case there is no confusion, we will omit $\lambda$ from $m_{i}(\lambda)$ to simplify our notation. If $\lambda=\left(1^{m_{1}(\lambda)}, 2^{m_{2}(\lambda)}, 3^{m_{3}(\lambda)}, \ldots, n^{m_{n}(\lambda)}\right)$ is a partition of $n$ then $\sum_{i=1}^{n} i m_{i}(\lambda)=n$. We will dismiss $i^{m_{i}(\lambda)}$ from $\lambda$ when $m_{i}(\lambda)=0$. For example, we will write $\lambda=\left(1^{2}, 3,6^{2}\right)$ instead of $\lambda=\left(1^{2}, 2^{0}, 3,4^{0}, 5^{0}, 6^{2}, 7^{0}\right)$. If $\lambda$ and $\delta$ are two partitions we define the union $\lambda \cup \delta$ and subtraction $\lambda \backslash \delta$ (if exists) as the following partitions:

$$
\begin{gathered}
\lambda \cup \delta=\left(1^{m_{1}(\lambda)+m_{1}(\delta)}, 2^{m_{2}(\lambda)+m_{2}(\delta)}, 3^{m_{3}(\lambda)+m_{3}(\delta)}, \ldots\right) . \\
\lambda \backslash \delta=\left(1^{m_{1}(\lambda)-m_{1}(\delta)}, 2^{m_{2}(\lambda)-m_{2}(\delta)}, 3^{m_{3}(\lambda)-m_{3}(\delta)}, \ldots\right) \text { if } m_{i}(\lambda) \geq m_{i}(\delta) \text { for any } i .
\end{gathered}
$$

The cycle-type of a permutation of $\mathcal{S}_{n}$ is the partition of $n$ obtained from the lengthes of the cycles that appear in its decomposition into product of disjoint cycles. For example, the permutation

$$
(2,4,1,6)(3,8,10,12)(5)(7,9,11)
$$

of $\mathcal{S}_{12}$ has cycle-type $\left(1,3,4^{2}\right)$. In this paper we will denote the cycle-type of a permutation $\omega$ by $\operatorname{ct}(\omega)$. It is well known that two permutations of $\mathcal{S}_{n}$ belong to the same conjugacy class if and only if they have the same cycle-type. Thus the conjugacy classes of the Symmetric group $\mathcal{S}_{n}$ can be indexed by partitions of $n$. If $\lambda=\left(1^{m_{1}(\lambda)}, 2^{m_{2}(\lambda)}, 3^{m_{3}(\lambda)}, \ldots, n^{m_{n}(\lambda)}\right)$ is a partition of $n$, we will denote by $C_{\lambda}$ the conjugacy class of $\mathcal{S}_{n}$ associated to $\lambda$ :

$$
C_{\lambda}:=\left\{\sigma \in \mathcal{S}_{n} \mid \operatorname{ct}(\sigma)=\lambda\right\}
$$

The cardinal of $C_{\lambda}$ is given by:

$$
\left|C_{\lambda}\right|=\frac{n !}{z_{\lambda}}
$$


where

$$
z_{\lambda}=1^{m_{1}(\lambda)} m_{1}(\lambda) ! 2^{m_{2}(\lambda)} m_{2}(\lambda) ! \cdots n^{m_{n}(\lambda)} m_{n}(\lambda) !
$$

The family $\left(\mathcal{C}_{\rho}\right)_{\rho \vdash n}$, indexed by partitions of $n$ where $\mathcal{C}_{\lambda}$ is the sum of permutations of $\mathcal{S}_{n}$ with cycle-type $\rho$

$$
\mathcal{C}_{\rho}=\sum_{\sigma \in C_{\rho}} \sigma
$$

forms a basis for the center of the symmetric group algebra. If $\lambda$ and $\delta$ are two partitions of $n$, the product $\mathcal{C}_{\lambda} \mathcal{C}_{\delta}$ can be written as a linear combination of the elements $\left(\mathcal{C}_{\rho}\right)_{\rho \vdash n}$ as follows:

$$
\mathcal{C}_{\lambda} \mathcal{C}_{\delta}=\sum_{\rho \vdash n} c_{\lambda \delta}^{\rho} \mathcal{C}_{\rho}
$$

The coefficients $c_{\lambda \delta}^{\rho}$ are called the structure coefficients of the center of the Symmetric group algebra. There is a combinatorial way to find these structure coefficients. To compute $c_{\lambda \delta}^{\rho}$, we fix a permutation $\gamma \in C_{\rho}$, that means $\gamma$ is a permutation of $\mathcal{S}_{n}$ with $\operatorname{ct}(\gamma)=\rho$, then $c_{\lambda \delta}^{\rho}$ is the cardinal of the followin set:

$$
c_{\lambda \delta}^{\rho}=\mid\left\{(\alpha, \beta) \in C_{\lambda} \times C_{\delta} \text { such that } \alpha \circ \beta=\gamma\right\} \mid .
$$

Alternatively,

$$
c_{\lambda \delta}^{\rho}=\mid\left\{\beta \in C_{\delta} \text { such that } \operatorname{ct}\left(\gamma \circ \beta^{-1}\right)=\lambda\right\} \mid .
$$

We will use the above two formulas (1) and (2) to compute the coefficients $c_{\lambda \delta}^{\rho}$ in this paper. Since there is no general formula for the structure coefficients of $Z\left(\mathbb{C}\left[\mathcal{S}_{n}\right]\right)$, the combinatorial way is useful to investigate a formula in case of special partitions. For example, to compute the product $\mathcal{C}_{\left(1^{n-2}, 2\right)} \mathcal{C}_{\left(1^{n-2}, 2\right)}$, we need to know how a transposition acts on another transposition. The composite of two transpositions of $\mathcal{S}_{n}$ gives:

- the identity if both transpositions are equals,

- a cycle of length three if they share only one element in their support,

- a product of two transpositions if their support are disjoint.

By using the combinatorial way, see [12, Example 6.5],we deduce that:

$$
\mathcal{C}_{\left(1^{n-2}, 2\right)} \mathcal{C}_{\left(1^{n-2}, 2\right)}=\frac{n(n-1)}{2} \mathcal{C}_{\left(1^{n}\right)}+3 \mathcal{C}_{\left(1^{n-3}, 3\right)}+2 \mathcal{C}_{\left(1^{n-4}, 2^{2}\right)}
$$

In fact, if $\lambda$ is a partition of $n$, there exists an explicit formula for the product $\mathcal{C}_{\lambda} \mathcal{C}_{\left(1^{n-2}, 2\right)}$ of the class corresponding to $\lambda$ with the class of transpositions. It appears for the first time in [8] and as stated here it can be found in [11, Proposition 2.8].

Proposition 2.1. Let $\lambda=\left(1^{m_{1}}, 2^{m_{2}}, \ldots, n^{m_{n}}\right)$ be any partition of $n$ then:

$$
\mathcal{C}_{\lambda} \cdot \mathcal{C}_{\left(1^{n-2}, 2\right)}=\sum_{i=2, m_{i} \neq 0}^{n} \sum_{k \leq E(i / 2)} \phi_{i}^{k} \mathcal{C}_{\lambda_{i}^{k}}+\sum_{i=1, m_{i} \geq 2}^{n} \phi_{i}^{\prime}{ }^{i} \mathcal{C}_{\lambda_{i}^{\prime} i}+\sum_{i=1, m_{i} \neq 0}^{n} \sum_{k>i, m_{k} \neq 0}^{n-i} \phi_{i}^{\prime k} \mathcal{C}_{\lambda_{i}^{\prime} k}
$$


Where

$$
\begin{gathered}
\lambda_{i}^{k}= \begin{cases}\lambda \cup(k, i-k) \backslash(i) & \text { if } \quad i-k \neq k \\
\lambda \cup\left(k^{2}\right) \backslash(i) & \text { if } \quad i-k=k\end{cases} \\
\lambda_{i}^{\prime k}=\left\{\begin{array}{l}
\lambda \backslash(i, k) \cup(i+k) \\
\lambda \backslash\left(i^{2}\right) \cup(2 i)
\end{array}\right. \\
\phi_{i}^{k}= \begin{cases}k\left(m_{k}+1\right)(i-k)\left(m_{i-k}+1\right) & \text { if } k \neq i \\
\frac{k^{2}\left(m_{k}+1\right)\left(m_{k}+2\right)}{2} & \text { if } i-k \neq k\end{cases} \\
\phi_{i}^{\prime k}= \begin{cases}(i+k)\left(m_{i+k}+1\right) & \text { if } i-k=k \\
i\left(m_{2 i}+1\right) & \text { if } k=i\end{cases}
\end{gathered}
$$

Example 2.2. 1. When $\lambda=\left(1^{n-2}, 2\right)$, we get:

$$
C_{\left(1^{n-2}, 2\right)} \mathcal{C}_{\left(1^{n-2}, 2\right)}=\phi_{2}^{1} \mathcal{C}_{\lambda_{2}^{1}}+\phi_{1}^{\prime} \mathcal{C}_{\lambda_{1}^{\prime 1}}+\phi_{1}^{\prime 2} \mathcal{C}_{\lambda_{1}^{\prime 2}}=\frac{n(n-1)}{2} \mathcal{C}_{\left(1^{n}\right)}+2 \mathcal{C}_{\left(1^{n-4}, 2^{2}\right)}+3 \mathcal{C}_{\left(1^{n-3}, 3\right)}
$$

2. If $r \geq 2$ and $n \geq 2 r+2$, we have:

$$
\begin{aligned}
\mathcal{C}_{\left(1^{n-2 r}, 2 r\right)} \cdot \mathcal{C}_{\left(1^{n-2}, 2\right)} & =\sum_{1 \leq k \leq r} \phi_{2 r}^{k} \mathcal{C}_{\lambda_{2 r}^{k}}+\phi_{1}^{\prime 1} \mathcal{C}_{\lambda_{1}^{\prime 1}}+\phi_{1}^{\prime 2 r} \mathcal{C}_{\lambda_{1}^{\prime 2 r}} \\
& =(n-2 r+1)(2 r-1) \mathcal{C}_{\left(1^{n-2 r+1}, 2 r-1\right)}+\sum_{1<k<r} k(2 r-k) \mathcal{C}_{\left(1^{n-2 r}, k, 2 r-k\right)} \\
& +r^{2} \mathcal{C}_{\left(1^{n-2 r}, r^{2}\right)}+\mathcal{C}_{\left(1^{n-2 r-2}, 2,2 r\right)}+(1+2 r) \mathcal{C}_{\left(1^{n-2 r-1}, 2 r+1\right)}
\end{aligned}
$$

3. If $r \geq 1$ and $n \geq 2 r+3$, we have:

$$
\begin{aligned}
\mathcal{C}_{\left(1^{n-2 r-1}, 2 r+1\right)} \cdot \mathcal{C}_{\left(1^{n-2}, 2\right)} & =\sum_{1 \leq k \leq r} \phi_{2 r+1}^{k} \mathcal{C}_{\lambda_{2 r+1}^{k}}+\phi_{1}^{\prime} \mathcal{C}_{\lambda_{1}^{\prime 1}}+\phi_{1}{ }^{2 r+1} \mathcal{C}_{\lambda_{1}^{\prime 2 r+1}} \\
& =(n-2 r)(2 r) \mathcal{C}_{\left(1^{n-2 r}, 2 r\right)}+\sum_{1<k<r} k(2 r+1-k) \mathcal{C}_{\left(1^{n-2 r-1}, k, 2 r+1-k\right)} \\
& +\mathcal{C}_{\left(1^{n-2 r-3}, 2,2 r+1\right)}+(2+2 r) \mathcal{C}_{\left(1^{n-2 r-2}, 2 r+2\right)},
\end{aligned}
$$

4. The above proposition along with the derived formulas in items (1), (2) and (3) give the following expressions in case $n=6$ :

$$
\begin{aligned}
\mathcal{C}_{\left(1^{4}, 2\right)} \cdot \mathcal{C}_{\left(1^{4}, 2\right)} & =15 \mathcal{C}_{\left(1^{n}\right)}+2 \mathcal{C}_{\left(1^{2}, 2^{2}\right)}+3 \mathcal{C}_{\left(1^{3}, 3\right)} \\
\mathcal{C}_{\left(1^{3}, 3\right)} \cdot \mathcal{C}_{\left(1^{4}, 2\right)} & =8 \mathcal{C}_{\left(1^{4}, 2\right)}+\mathcal{C}_{(1,2,3)}+4 \mathcal{C}_{\left(1^{2}, 4\right)} \\
\mathcal{C}_{(1,5)} \cdot \mathcal{C}_{\left(1^{4}, 2\right)} & =8 \mathcal{C}_{\left(1^{2}, 4\right)}+6 \mathcal{C}_{(6)} \\
\mathcal{C}_{\left(1^{2}, 4\right)} \cdot \mathcal{C}_{\left(1^{4}, 2\right)} & =9 \mathcal{C}_{\left(1^{3}, 3\right)}+4 \mathcal{C}_{\left(1^{2}, 2^{2}\right)}+\mathcal{C}_{(2,4)}+5 \mathcal{C}_{(1,5)} \\
\mathcal{C}_{(6)} \cdot \mathcal{C}_{\left(1^{4}, 2\right)} & =5 \mathcal{C}_{(1,5)}+8 \mathcal{C}_{(2,4)}+9 \mathcal{C}_{\left(3^{2}\right)} \\
\mathcal{C}_{\left(2^{3}\right)} \cdot \mathcal{C}_{\left(1^{4}, 2\right)} & =\phi_{2}^{1} \mathcal{C}_{\lambda_{2}^{1}}+\phi_{2}^{\prime 2} \mathcal{C}_{\lambda_{2}^{\prime 2}}=\mathcal{C}_{\left(1^{2}, 2^{2}\right)}+2 \mathcal{C}_{(2,4)} \\
\mathcal{C}_{\left(3^{2}\right)} \cdot \mathcal{C}_{\left(1^{4}, 2\right)} & =\phi_{3}^{1} \mathcal{C}_{\lambda_{3}^{1}}+\phi_{3}^{\prime 3} \mathcal{C}_{\lambda_{3}^{\prime 3}}=2 \mathcal{C}_{(1,2,3)}+3 \mathcal{C}_{(6)} \\
C_{\left(1^{2}, 2^{2}\right)} \cdot \mathcal{C}_{\left(1^{4}, 2\right)} & =\phi_{2}^{1} \mathcal{C}_{\lambda_{2}^{1}}+\phi_{1}^{\prime 1} \mathcal{C}_{\lambda_{1}^{\prime 1}}+\phi_{2}^{\prime 2} \mathcal{C}_{\lambda_{2}^{\prime 2}}+\phi_{1}^{\prime 2} \mathcal{C}_{\lambda_{1}^{\prime 2}}=6 \mathcal{C}_{\left(1^{4}, 2\right)}+3 \mathcal{C}_{\left(2^{3}\right)}+2 \mathcal{C}_{\left(1^{2}, 4\right)}+3 \mathcal{C}_{(1,2,3)} \\
\mathcal{C}_{(1,2,3)} \cdot \mathcal{C}_{\left(1^{4}, 2\right)} & =\phi_{2}^{1} \mathcal{C}_{\lambda_{2}^{1}}+\phi_{3}^{1} \mathcal{C}_{\lambda_{3}^{1}}+\phi_{1}^{\prime 2} \mathcal{C}_{\lambda_{1}^{\prime 2}}+\phi_{1}^{\prime 3} \mathcal{C}_{\lambda_{1}^{\prime 3}}+\phi_{2}^{\prime 3} \mathcal{C}_{\lambda_{2}^{\prime 3}} \\
& =3 \mathcal{C}_{\left(1^{3}, 3\right)}+8 \mathcal{C}_{\left(1^{2}, 2^{2}\right)}+6 \mathcal{C}_{\left(3^{2}\right)}+4 \mathcal{C}_{(2,4)}+5 \mathcal{C}_{(1,5)} \\
\mathcal{C}_{(2,4)} \cdot \mathcal{C}_{\left(1^{4}, 2\right)} & =\phi_{2}^{1} \mathcal{C}_{\lambda_{2}^{1}}+\phi_{4}^{1} \mathcal{C}_{\lambda_{4}^{1}}+\phi_{4}^{2} \mathcal{C}_{\lambda_{4}^{2}}+\phi_{2}^{\prime 4} \mathcal{C}_{\lambda_{2}^{\prime 4}}=\mathcal{C}_{\left(1^{2}, 4\right)}+3 \mathcal{C}_{(1,2,3)}+12 \mathcal{C}_{\left(2^{3}\right)}+6 \mathcal{C}_{(6)}
\end{aligned}
$$




\section{Some new explicit formulas for the structure coefficients}

In this section we give new explicit formulas for the structure coefficients of the center of the symmetric group algebra involving cycles of length three. Specifically, in Proposition 3.4 we give an explicit formula for the coefficients $c_{\lambda,\left(1^{n-3}, 3\right)}^{\lambda}$ and in Proposition 3.10 the complete expression of the the product $\mathcal{C}_{\left(1^{n-k}, k\right)} \mathcal{C}_{\left(1^{n-3}, 3\right)}$ is given. We hope that these two results will help find an explicit expression for the product of an arbitrary class of permutations with the class of cycles of length three similar to that given in Proposition 2.1.

A cycle of length $k$ will be sometimes called a $k$-cycle. If $f$ and $g$ are two permutations we will write $f g$ from time to time to denote the composite $f \circ g$. Occasionally and to simplify our notation, we will dismiss the fixed elements when writting a permutation as a product of disjoint cycles.

Let $\lambda=\left(1^{m_{1}}, 2^{m_{2}}, \ldots, n^{m_{n}}\right)$ be a partition of $n$. The following permutation of $n$

$$
p_{\lambda}:=\underbrace{(1,2)(3,4) \cdots\left(2 m_{2}-1,2 m_{2}\right)}_{m_{2}(\lambda) \text { transposition }} \underbrace{\left(2 m_{2}+1,2 m_{2}+2,2 m_{2}+3\right) \cdots\left(2 m_{2}+1,2 m_{2}+2,2 m_{2}+3\right)}_{m_{3}(\lambda) \text { cycles of length } 3} \cdots
$$

will be called the canonical permutation of $n$ associated to $\lambda$. For example:

$$
\begin{gathered}
p_{\left(2^{2}, 3\right)}=(1,2)(3,4)(5,6,7) \\
p_{\left(1^{3}, 4^{2}, 5\right)}=(1,2,3,4)(5,6,7,8)(9,10,11,12,13)(14)(15)(16) .
\end{gathered}
$$

Lemma 3.1. Let $n$ and $k$ be integers such that $n \geq k \geq 3$, then

$$
\operatorname{Card}\left(\left\{(\alpha, \beta) \in C_{\left(1^{n-k}, k\right)} \times C_{\left(1^{n-3}, 3\right)} \text { such that } \alpha \circ \beta=p_{\left(1^{n-k}, k\right)}\right\}\right)=\left(\begin{array}{l}
k \\
3
\end{array}\right) .
$$

Lemma 3.2. Let $n$ and $k$ be integers such that $n>k \geq 2$, then

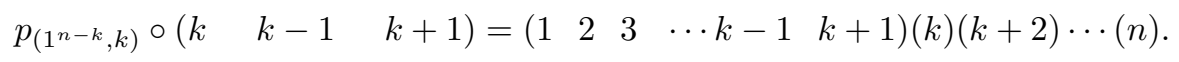

Lemma 3.3. Let $\left(i_{1}, i_{2}, \cdots, i_{k}\right)$ and $\left(r_{1}, r_{2}, \cdots, r_{t}\right)$ be two disjoint cycles of size $k$ and $t$ respectively with $t>k \geq 2$, then:

$$
\left(i_{1}, i_{2}, \cdots, i_{k}\right)\left(r_{1}, r_{2}, \cdots, r_{t}\right)\left(i_{k}, r_{t}, r_{t-k}\right)=\left(i_{1}, i_{2}, \cdots, i_{k}, r_{1}, r_{2}, \cdots, r_{t-k}\right)\left(r_{t-k+1}, \cdots, r_{t}\right) .
$$

Proposition 3.4. Let $\lambda=\left(1^{m_{1}}, 2^{m_{2}}, \ldots, n^{m_{n}}\right)$ be any partition of $n \geq 3$ then:

$$
c_{\lambda\left(1^{n-3}, 3\right)}^{\lambda}=\sum_{\substack{i \geq 3, m_{i} \geq 1}} m_{i}\left(\begin{array}{l}
i \\
3
\end{array}\right)+\sum_{\substack{i \geq 2, m_{i} \geq 1}} m_{1} \cdot i \cdot m_{i}+\sum_{\substack{i \geq 2, m_{i} \geq 1}} \sum_{\substack{j>i \\
m_{j} \geq 1}} i \cdot j \cdot m_{i} m_{j} .
$$

Proof. To compute $c_{\lambda\left(1^{n-3}, 3\right)}^{\lambda}$ by the combinatorial way, we should find the number of couples $(\alpha, \beta)$ such that $\operatorname{ct}(\alpha)=\lambda, \operatorname{ct}(\beta)=\left(1^{n-3}, 3\right)$ and $\alpha \beta=p_{\lambda}$. We have to consider the following three cases:

1. There exists $i \geq 3$ such that $m_{i} \neq 0$, say $\mathcal{C}$ is a cycle of length $i$ in $p_{\lambda}$. We will choose the three elements of the 3 -cycle in $\beta$ among the elements of the cycle $\mathcal{C}$ and thus $\alpha$ will act as $p_{\lambda}$ outside the support of $\mathcal{C}$. By Lemma 3.1, and after renormalisation we can find $\left(\begin{array}{l}i \\ 3\end{array}\right)$ such couples $(\alpha, \beta)$. We can repeat the same process as many times as we have cycles of length $i$ in $\lambda$ which justify the first coefficient

$$
\sum_{\substack{i \geq 3, m_{i} \geq 1}} m_{i}\left(\begin{array}{l}
i \\
3
\end{array}\right)
$$

in the formula (3). 
2. There exists $i \geq 2$ such that $m_{i} \neq 0$ and $m_{1} \neq 0$. Consider $\left(a_{1}, a_{2}, \cdots, a_{i}\right)$ to be a cycle of length $i$ in $p_{\lambda}$. Choose $\alpha$ to be the same as $p_{\lambda}$ except that the cycle $\left(a_{1}, a_{2}, \cdots, a_{i}\right)$ is replaced by $\left(a_{1}, a_{2}, \cdots, a_{i-1}, b\right)$ where $b$ is a chosen element among the $m_{1}$ unchaged integer by $p_{\lambda}$. Then by Lemme $3.2, \alpha \circ \beta=p_{\lambda}$, where $\beta=\left(a_{i-1}, a_{i}, b\right)$. Since there exists $i$ way to write the cycle $\left(a_{1}, a_{2}, \cdots, a_{i-1}, b\right)$ and for each way the corresponding $\beta$ is different, we conclude that for this case there are

$$
\sum_{\substack{i \geq 2, m_{i} \geq 1}} m_{1} \cdot i \cdot m_{i}
$$

ways to choose the couple $(\alpha, \beta)$.

3. There exists $i \geq 2$ and $j>i$ such that $m_{i} \neq 0$ and $m_{j} \neq 0$. Suppose $\left(a_{1}, a_{2}, \cdots, a_{i}\right)$ and $\left(b_{1}, b_{2}, \cdots, b_{j}\right)$ are two cycles of length $i$ and $j$ in $p_{\lambda}$. Then by Lemma 3.3, if we take $\alpha$ to be the same as $p_{\lambda}$ except that the cycles $\left(a_{1}, a_{2}, \cdots, a_{i}\right)$ and $\left(b_{1}, b_{2}, \cdots, b_{j}\right)$ are replaced by $\left(b_{i+1}, b_{i+2}, \cdots, b_{j}, a_{1}, a_{2}, \cdots, a_{i}\right)$ and $\left(b_{1}, \cdots, b_{i}\right)$. Then it is clear that $\operatorname{ct}(\alpha)=\lambda$ and $\alpha \beta=p_{\lambda}$ where $\beta=\left(b_{i}, a_{i}, b_{j}\right)$. Since there are $j$ ways to write the cycle $\left(b_{i+1}, b_{i+2}, \cdots, b_{j}, a_{1}, a_{2}, \cdots, a_{i}\right)$ and $i$ ways to write $\left(b_{1}, \cdots, b_{i}\right)$, we will get in total $i j$ couples $(\alpha, \beta)$ satisfying the desired conditions. We can repeat the same process as many times as we have cycles of lengthes $i$ and $j$ in $p_{\lambda}$ which gives reason to the coefficient

$$
\sum_{\substack{i \geq 2, m_{i} \geq 1}} \sum_{\substack{j>i, m_{j} \geq 1}} i \cdot j \cdot m_{i} m_{j}
$$

that appears in formula (3).

Example 3.5. We illustrate the above proof by an example. Suppose $\lambda=\left(1^{2}, 2,4\right)$ is a partition of 8 . We will list below all the possible permutations $\alpha$ and $\beta$ of 8 such that $\operatorname{ct}(\alpha)=\lambda, \operatorname{ct}(\beta)=\left(1^{5}, 3\right)$ and $\alpha \circ \beta=p_{\lambda}$. There are three cases to distinguish as in the above proof:

a. The support of $\beta$ is taken from the cycle of length 4 of $p_{\lambda}$ :

$$
\begin{aligned}
& \text { 1. } \alpha=(1,2)(3,5,4,6) \text { and } \beta=(3,5,4) \\
& \text { 2. } \alpha=(1,2)(3,6,4,5) \text { and } \beta=(3,6,5) \\
& \text { 3. } \alpha=(1,2)(4,6,5,3) \text { and } \beta=(4,6,5) \\
& \text { 4. } \alpha=(1,2)(5,6,4,3) \text { and } \beta=(3,6,4)
\end{aligned}
$$

b. Two elements in the support of $\beta$ are taken either from the cycle of length 2 or the cycle of lentgh 4 of $p_{\lambda}$ and the third one is taken among the invariant elements of $p_{\lambda}$ :

$$
\begin{aligned}
\text { 1. } \alpha=(1,7)(3,4,5,6) \text { and } \beta=(1,2,7) \\
\text { 2. } \alpha=(1,8)(3,4,5,6) \text { and } \beta=(1,2,8) \\
\text { 3. } \alpha=(2,7)(3,4,5,6) \text { and } \beta=(2,1,7) \\
\text { 4. } \alpha=(2,8)(3,4,5,6) \text { and } \beta=(2,1,8) \\
\text { 5. } \alpha=(1,2)(3,4,5,7) \text { and } \beta=(7,5,6) \\
\text { 6. } \alpha=(1,2)(3,4,5,8) \text { and } \beta=(8,5,6) \\
\text { 7. } \alpha=(1,2)(3,4,7,6) \text { and } \beta=(4,5,7) \\
\text { 8. } \alpha=(1,2)(3,4,8,6) \text { and } \beta=(4,5,8) \\
\text { 9. } \alpha=(1,2)(3,7,5,6) \text { and } \beta=(3,4,7) \\
\text { 10. } \alpha=(1,2)(3,8,5,6) \text { and } \beta=(3,4,8)
\end{aligned}
$$


11. $\alpha=(1,2)(7,4,5,6)$ and $\beta=(3,7,6)$

12. $\alpha=(1,2)(8,4,5,6)$ and $\beta=(3,8,6)$

b. Two elements in the support of $\beta$ are taken from the cycle of length 4 of $p_{\lambda}$ and the third one is taken from the cycle of length 2 :

1. $\alpha=(3,4)(5,6,1,2)$ and $\beta=(2,6,4)$

2. $\alpha=(3,4)(5,6,2,1)$ and $\beta=(1,6,4)$

3. $\alpha=(6,3)(4,5,1,2)$ and $\beta=(2,5,3)$

4. $\alpha=(6,3)(4,5,2,1)$ and $\beta=(1,5,3)$

5. $\alpha=(5,6)(3,4,1,2)$ and $\beta=(2,4,6)$

6. $\alpha=(5,6)(3,4,2,1)$ and $\beta=(1,4,6)$

7. $\alpha=(4,5)(6,3,1,2)$ and $\beta=(2,3,5)$

8. $\alpha=(4,5)(6,3,2,1)$ and $\beta=(1,3,5)$

Thus $\left[\mathcal{C}_{\left(1^{2}, 2,4\right)}\right] \mathcal{C}_{\left(1^{2}, 2,4\right)} \mathcal{C}_{\left(1^{5}, 3\right)}=4+12+8=24$.

Example 3.6. By formula (3), we get:

$$
\begin{gathered}
c_{(2,6),\left(1^{5}, 3\right)}^{(2,6)}=32, \quad c_{\left(1,4^{2}\right),\left(1^{6}, 3\right)}^{\left(1,4^{2}\right)}=16, \quad c_{(2,7),\left(1^{6}, 3\right)}^{(2,7)}=49, \quad c_{(3,6),\left(1^{6}, 3\right)}^{(3,6)}=39, \quad c_{\left(1^{4}, 5\right),\left(1^{6}, 3\right)}^{\left(1^{4}, 5\right)}=30, \\
c_{\left(1^{2}, 2,5\right),\left(1^{6}, 3\right)}^{\left(1^{2}, 2,5\right)}=34, \quad c_{(2,3,4),\left(1^{6}, 3\right)}^{(2,3,4)}=31, \quad c_{\left(1,2,3^{2}\right),\left(1^{6}, 3\right)}^{\left(1,2,2^{2}\right)}=22, \quad c_{\left(1^{3}, 2,4\right),\left(1^{6}, 3\right)}^{\left(1^{3}, 2,4\right)}=30, \\
c_{\left(1^{2}, 2,3^{2}\right),\left(1^{7}, 3\right)}^{\left(1^{2}, 23^{2}\right)}=30, \quad c_{\left(1^{2}, 2,6\right),\left(1^{7}, 3\right)}^{\left(1^{2}, 2,6\right)}=48, \quad c_{(3,7),\left(1^{7}, 3\right)}^{(3,7)}=57 .
\end{gathered}
$$

We used the SageMath software [10] to confirm these results.

We turn now to compute remarkable coefficients in the product $\mathcal{C}_{\lambda} \mathcal{C}_{\left(1^{n-3}, 3\right)}$, the coefficients $c_{\lambda,\left(1^{n-3}, 3\right)}^{\left(1^{n-3}, 3\right)}$, $c_{\lambda,\left(1^{n-3}, 3\right)}^{\lambda \backslash\left(1^{3}\right) \cup(3)}$ and $c_{\lambda,\left(11^{n-3}, 3\right)}^{\lambda \backslash(3) \cup\left(1^{3}\right)}$.

Proposition 3.7. Let $\lambda=\left(1^{m_{1}}, 2^{m_{2}}, \ldots, n^{m_{n}}\right)$ be any partition of $n \geq 6$ then:

$$
c_{\lambda\left(1^{n-3}, 3\right)}^{\left(1^{n-3}, 3\right)}= \begin{cases}2\left(\begin{array}{c}
n-3 \\
3
\end{array}\right) & \text { if } \lambda=\left(1^{n-6}, 3^{2}\right), \\
3 m_{1}+1 & \text { if } \lambda=\left(1^{n-3}, 3\right), \\
0 & \text { otherwise. }\end{cases}
$$

Proof. First if $\lambda=\left(1^{n-3}, 3\right)$, use formula (3) to get the result. Suppose now that $\lambda=\left(1^{n-6}, 3^{2}\right)$, then $c_{\lambda\left(1^{n-3}, 3\right)}^{\left(1^{n-3}, 3\right)}$ is the number of couples $(\alpha, \beta)$ such that $\operatorname{ct}(\alpha)=\left(1^{n-6}, 3^{2}\right), \operatorname{ct}(\beta)=\left(1^{n-3}, 3\right)$ and $\alpha \beta=p_{\left(1^{n-3}, 3\right)}$. To choose $\alpha$, pick the first 3-cycle of $\alpha$ to be $(1,2,3)$ and form the second 3 -cycle $\mathcal{C}$ by choosing arbitrary 3 integers of the remaining $n-3$ integers, there are $2\left(\begin{array}{c}n-3 \\ 3\end{array}\right)$ such way to do that. Take $\beta=\mathcal{C}^{-1}$, then $\alpha \beta=p_{\left(1^{n-3}, 3\right)}$ and $c_{\left(1^{n-6}, 3^{2}\right),\left(1^{n-3}, 3\right)}^{\left(1^{n-3}, 3\right)}=2\left(\begin{array}{c}n-3 \\ 3\end{array}\right)$.

Proposition 3.8. Let $\lambda=\left(1^{m_{1}}, 2^{m_{2}}, \ldots, n^{m_{n}}\right)$ be any partition of $n \geq 3$ such that $m_{1} \geq 3$ then:

$$
c_{\lambda\left(1^{n-3}, 3\right)}^{\lambda \backslash\left(1^{3}\right) \cup(3)}=m_{3}+1 .
$$


Proof. Any permutation with cycle-type $\lambda \backslash\left(1^{3}\right) \cup(3)$ has $m_{3}+1$ cycle of length 3 . Let $\gamma$ be such a permutation. The coefficient $c_{\lambda,\left(1^{n-3}, 3\right)}^{\lambda \backslash\left(1^{3}\right) \cup(3)}$ is the number of couples $(\alpha, \beta)$ such that $\operatorname{ct}(\alpha)=\lambda$, $\operatorname{ct}(\beta)=$ $\left(1^{n-3}, 3\right)$ and $\alpha \beta=\gamma$. We can choose $\beta$ to be any 3 cycle among the $m_{3}+13$-cycles of $\gamma$ and $\alpha$ is the same as $\gamma$ except that the cycle $\beta$ is replaced by the identity on its support.

Proposition 3.9. Let $\lambda=\left(1^{m_{1}}, 2^{m_{2}}, \ldots, n^{m_{n}}\right)$ be any partition of $n \geq 3$ such that $m_{3} \geq 1$ then:

$$
c_{\lambda\left(1^{n-3}, 3\right)}^{\lambda \backslash(3) \cup\left(1^{3}\right)}=2\left(\begin{array}{c}
m_{1}+3 \\
3
\end{array}\right) .
$$

Proof. Let $\rho$ be a permutation such that $\operatorname{ct}(\rho)=\lambda \backslash(3) \cup\left(1^{3}\right)$. The coefficient $c_{\lambda,\left(1^{n-3}, 3\right)}^{\lambda \backslash(3) \cup\left(1^{3}\right)}$ is the number of couples $(\alpha, \beta)$ such that $\operatorname{ct}(\alpha)=\lambda, \operatorname{ct}(\beta)=\left(1^{n-3}, 3\right)$ and $\alpha \beta=\rho$. The permutation $\rho$ has $m_{1}+3$ fixed element. Let $\beta$ be any 3 -cycle formed with these $m_{1}+3$ fixed elements. There are $2\left(\begin{array}{c}m_{1}+3 \\ 3\end{array}\right)$ way to make $\beta$. Since $\beta$ is a 3 -cycle, its inverse $\beta^{-1}$ is a 3 -cycle also and $\rho \beta^{-1}$ has cycle-type $\lambda$.

The next proposition gives an explicit expression for the product of the class of 3-cycles by the class of cycles of length $k$ for an arbitrary $k$.

Proposition 3.10. Let $n \geq k>3$ then:

$$
\begin{aligned}
& \mathcal{C}_{\left(1^{n-k}, k\right)} \mathcal{C}_{\left(1^{n-3}, 3\right)}=\sum_{\substack{i \leq j \leq r, i+j+r=k}} 2 i j r\left(\begin{array}{c}
m_{1}\left(\left(1^{n-k}\right) \cup(i, j, r)\right) \\
n-k
\end{array}\right) \mathcal{C}_{\left(1^{n-k}\right) \cup(i, j, r)} \\
& +\underbrace{\sum_{i=2}^{[(k+1) / 2]} 2 i(k+1-i) \mathcal{C}_{\left(1^{n-k-1}\right) \cup(i, k+1-i)}}_{\text {if } n \geq k+1}+\underbrace{(k+2) \mathcal{C}_{\left(1^{n-k-2}\right) \cup(k+2)}}_{\text {if } n \geq k+2} \\
& +\underbrace{\mathcal{C}_{\left(1^{n-k-3}, k\right) \cup(3)}}_{\text {if } n \geq k+3}+\left[\left(\begin{array}{l}
k \\
3
\end{array}\right)+(n-k) k\right] \mathcal{C}_{\left(1^{n-k}, k\right)}
\end{aligned}
$$

Proof. We use formula (2) in this proof. Let $i, j$ and $r$ be three integers such that $i \leq j \leq r$ and $i+j+r=k$. Consider the permutation $p_{\left(1^{n-k}\right) \cup(i, j, r)}$, the coefficient $c_{\left(1^{n-k}, k\right),\left(1^{n-3}, 3\right)}^{\left(1^{n-k}\right) \cup(i, r)}$ is the number of permutations $\beta$ such that $\operatorname{ct}(\beta)=\left(1^{n-3}, 3\right)$ and $\operatorname{ct}\left(p_{\left(1^{n-k}\right) \cup(i, j, r)} \beta^{-1}\right)=\left(1^{n-k}, k\right)$. Choose $\beta=(x, y, z)$ to be any cycle formed with an element $x$ of $[i]$, an element $y$ of $[i+j] \backslash[i]$ and an element $z$ of $[k] \backslash[i+j]$. There are thus $2 i j r$ ways to form $\beta$. Then $p_{\left(1^{n-k}\right) \cup(i, j, r)} \beta^{-1}$ is the following cycle of length $k$

$$
(1,2, \cdots, x, z+1, \cdots, k, i+j+1, \cdots, z, y+1, \cdots, i+j, i+1, \cdots, y, x+1, \cdots, i) .
$$

To obtain the coefficient $2 \operatorname{ijr}\left(\begin{array}{c}m_{1}\left(\left(1_{n-k}^{n-k}\right) \cup(i, j, r)\right) \\ n-k\end{array}\right)$ that appears in formula (4), one should remark that when $i=1, x$ can be chosen among the $m_{1}+1$ fixed elements of $p_{\left(1^{n-k}\right) \cup(i, j, r)}$, and when $i=1$ and $j=1, x$ and $y$ can be chosen among the $m_{1}+2$ fixed elements of $p_{\left(1^{n-k}\right) \cup(i, j, r)}$.

Now fix an integer $2 \leq i \leq[(k+1) / 2]$. To compute $c_{\left(1^{n-k}, k\right),\left(1^{n-3}, 3\right)}^{\left(1^{n-k-1}\right) \cup(i, k+1-i)}$, we need to determine the number of permutations $\beta$ such that $\operatorname{ct}(\beta)=\left(1^{n-3}, 3\right)$ and $\operatorname{ct}\left(p_{\left(1^{n-k-1}\right) \cup(i, k+1-i)} \beta^{-1}\right)=\left(1^{n-k}, k\right)$. We can choose $\beta$ to be:

- any 3 -cycle $(a, k, k+1)$ with $a \in[i]$, then

$$
\begin{aligned}
p_{\left(1^{n-k-1}\right) \cup(i, k+1-i)} \beta^{-1} & =(1,2, \cdots, i)(i+1, \cdots, k+1)(k+1, k, a)(k+2) \cdots(n) \\
& =(1,2, \cdots, a, i+1, \cdots, k, a+1, \cdots, i)(k+1)(k+2) \cdots(n) .
\end{aligned}
$$

Since there are $k-i+1$ ways to write the cycle $(i+1, \cdots, k+1)$, in total there are $i .(k-i+1)$ possibilities to choose $\beta$ in this case. 
- any 3 -cycle $(b, i-1, i)$ with $b \in[k] \backslash[i]$, then

$$
\begin{aligned}
p_{\left(1^{n-k-1}\right) \cup(i, k+1-i)} \beta^{-1} & =(1,2, \cdots, i)(i+1, \cdots, k+1)(i, i-1, b)(k+2) \cdots(n) \\
& =(1,2, \cdots, i-1, b+1, \cdots, k+1, i+1, \cdots, b)(k+2) \cdots(n) .
\end{aligned}
$$

Since there are $i$ ways to write the cycle $(1, \cdots, i)$, in total there are $i .(k-i+1)$ possibilities to choose $\beta$ in here.

Thus in total, we obtain the coefficient $2 i(k+1-i)$.

We turn now to calculate the number of permutations $\beta$ such that $\operatorname{ct}(\beta)=\left(1^{n-3}, 3\right)$ and $\operatorname{ct}\left(p_{\left(1^{n-k-2}\right) \cup(k+2)} \beta^{-1}\right)=\left(1^{n-k}, k\right)$. There are $k+2$ ways to choose $\beta$, it can be taken to be any cycle $(i, i+1, i+2)$ with $[i] \in[k+2](k+3 \equiv 1$ and $k+4 \equiv 2$ in here $)$. Then

$$
\begin{aligned}
p_{\left(1^{n-k-2}\right) \cup(k+2)} \beta^{-1} & =(1,2, \cdots, k+2)(i+2, i+1, i)(k+3) \cdots(n) \\
& =(1,2 \cdots, i, i+3, \cdots, k+2)(i+1)(i+2)(k+3) \cdots(n)
\end{aligned}
$$

is a $k$-cycle.

It remains to remark that the last two coefficients in formula (4) can be derived from Proposition 3.8 and Proposition 3.4 respectively.

Example 3.11. 1. We list below all the cases for $n=9$ :

$$
\begin{aligned}
& \mathcal{C}_{(9)} \mathcal{C}_{\left(1^{6}, 3\right)}= 14 \mathcal{C}_{\left(1^{2}, 7\right)}+24 \mathcal{C}_{(1,2,6)}+30 \mathcal{C}_{(1,3,5)}+40 \mathcal{C}_{\left(2^{2}, 5\right)}+32 \mathcal{C}_{\left(1,4^{2}\right)} \\
&+48 \mathcal{C}_{(2,3,4)}+54 \mathcal{C}_{\left(3^{3}\right)}+84 \mathcal{C}_{(9)} . \\
& \mathcal{C}_{(1,8)} \mathcal{C}_{\left(1^{6}, 3\right)}= 36 \mathcal{C}_{\left(1^{3}, 6\right)}+40 \mathcal{C}_{\left(1^{2}, 2,5\right)}+48 \mathcal{C}_{\left(1^{2}, 3,4\right)}+32 \mathcal{C}_{\left(1,2^{2}, 4\right)}+36 \mathcal{C}_{\left(1,2,3^{2}\right)} \\
&+40 \mathcal{C}_{(4,5)}+36 \mathcal{C}_{(3,6)}+28 \mathcal{C}_{(2,7)}+64 \mathcal{C}_{(1,8)} \cdot \\
& \mathcal{C}_{\left(1^{2}, 7\right)} \mathcal{C}_{\left(1^{6}, 3\right)}= 60 \mathcal{C}_{\left(1^{4}, 5\right)}+48 \mathcal{C}_{\left(1^{3}, 2,4\right)}+24 \mathcal{C}_{\left(1^{2}, 2^{2}, 3\right)}+54 \mathcal{C}_{\left(1^{3}, 3^{2}\right)}+32 \mathcal{C}_{\left(1,4^{2}\right)} \\
&+30 \mathcal{C}_{(1,3,5)}+24 \mathcal{C}_{(1,2,6)}+49 \mathcal{C}_{\left(1^{2}, 7\right)}+9 \mathcal{C}_{(9)} \cdot \\
& \mathcal{C}_{\left(1^{3}, 6\right)} \mathcal{C}_{\left(1^{6}, 3\right)}= 80 \mathcal{C}_{\left(1^{5}, 4\right)}+48 \mathcal{C}_{\left(1^{4}, 2,3\right)}+16 \mathcal{C}_{\left(1^{3}, 2^{3}\right)}+20 \mathcal{C}_{\left(1^{2}, 2,5\right)}+24 \mathcal{C}_{\left(1^{2}, 3,4\right)} \\
&+38 \mathcal{C}_{\left(1^{3}, 6\right)}+\mathcal{C}_{(3,6)}+8 \mathcal{C}_{(1,8)} \cdot \\
& \mathcal{C}_{\left(1^{4}, 5\right)} \mathcal{C}_{\left(1^{6}, 3\right)}= 90 \mathcal{C}_{\left(1^{6}, 3\right)}+40 \mathcal{C}_{\left(1^{5}, 2^{2}\right)}+7 \mathcal{C}_{\left(1^{2}, 7\right)}+16 \mathcal{C}_{\left(1^{3}, 2,4\right)}+18 \mathcal{C}_{\left(1^{3}, 3^{2}\right)} \\
&+\mathcal{C}_{(1,3,5)}+30 \mathcal{C}_{\left(1^{4}, 5\right)} . \\
& \mathcal{C}_{\left(1^{5}, 4\right)} \mathcal{C}_{\left(1^{6}, 3\right)}= 84 \mathcal{C}_{\left(1^{7}, 2\right)}+6 \mathcal{C}_{\left(1^{3}, 6\right)}+12 \mathcal{C}_{\left(1^{4}, 2,3\right)}+\mathcal{C}_{\left(1^{2}, 3,4\right)}+24 \mathcal{C}_{\left(1^{5}, 4\right)} . \\
& \mathcal{C}_{\left(1^{6}, 3\right)} \mathcal{C}_{\left(1^{6}, 3\right)=} 168 \mathcal{C}_{\left(1^{9}\right)}+5 \mathcal{C}_{\left(1^{4}, 5\right)}+8 \mathcal{C}_{\left(1^{5}, 2^{2}\right)}+2 \mathcal{C}_{\left(1^{3}, 3^{2}\right)}+19 \mathcal{C}_{\left(1^{6}, 3\right)} .
\end{aligned}
$$

2.

$$
\begin{aligned}
\mathcal{C}_{\left(1^{3}, 7\right)} \mathcal{C}_{\left(1^{7}, 3\right)} & =100 \mathcal{C}_{\left(1^{5}, 5\right)}+64 \mathcal{C}_{\left(1^{4}, 2,4\right)}+24 \mathcal{C}_{\left(1^{3}, 2^{2}, 3\right)}+72 \mathcal{C}_{\left(1^{4}, 3^{2}\right)}+32 \mathcal{C}_{\left(1^{2}, 4^{2}\right)} \\
& +30 \mathcal{C}_{\left(1^{2}, 3,5\right)}+24 \mathcal{C}_{\left(1^{2}, 2,6\right)}+\mathcal{C}_{(3,7)}+9 \mathcal{C}_{(1,9)}+56 \mathcal{C}_{\left(1^{3}, 7\right)}
\end{aligned}
$$

The coefficients $c_{\lambda \delta}^{\rho}$ can be expressed in terms of irreducible characters of the symmetric group:

$$
c_{\lambda \delta}^{\rho}=\frac{\left|\mathcal{C}_{\lambda}\right|\left|\mathcal{C}_{\delta}\right|}{n !} \sum_{\mu \vdash n} \frac{\chi_{\lambda}^{\mu} \chi_{\delta}^{\mu} \chi_{\rho}^{\mu}}{\operatorname{dim} V_{\mu}},
$$

where $\chi_{\lambda}^{\mu}$ is the irreducible character of the symmetric group associated to the partition $\mu$ evaluated on an element of the conjugacy class $\mathcal{C}_{\lambda}$ and $\operatorname{dim} V_{\mu}$ is the dimension of the irreducible representation associated to the partition $\mu$. The formula (4) is due to Frobenius. It was used along with the Murnaghan-Nakayama rule to compute the structure coefficients of the center of the symmetric group algebra, see [6] for the computation of the coefficients $c_{\lambda \mu}^{(n)}$. It will be interesting to investigate how the explicit formulas for the structure coefficients given in this paper could be found by the Frobenius formula. 


\section{References}

[1] Z. Arad, M. Herzog (Eds.), Products of Conjugacy Classes in Groups, Lecture Notes in Math., vol. 1112, Springer-Verlag, Berlin, 1985.

[2] E. A. Bertram, V. K. Wei, Decomposing a permutation into two large cycles: An enumeration, SIAM J. Algebraic Discrete Methods 1(4)(1980) 450-461.

[3] G. Boccara, Nombre de representations d'une permutation comme produit de deux cycles de longueurs donnees, Discrete Math. 29(2) (1980) 105-134.

[4] R. Cori, Un code pour les graphes planaires et ses applications, Astérisque 27 (1975) 169pp.

[5] H. K. Farahat, G. Higman, The centres of symmetric group rings, Proc. Roy. Soc. London Ser. A 250(1261) (1959) 212-221.

[6] A. Goupil, G. Schaeffer, Factoring n-cycles and counting maps of given genus, European J. Combin. 19(7) (1998) 819-834.

[7] D. M. Jackson, T. I. Visentin, Character theory and rooted maps in an orientable surface of given genus: Face-colored maps, Trans. Amer. Math. Soc. 322(1) (1990) 365-376.

[8] J. Katriel, J. Paldus, Explicit expression for the product of the class of transpositions with an arbitrary class of the symmetric group, R Gilmore (Ed.), Group Theoretical Methods in Physics, World Scientific, Singapore (1987) 503-506.

[9] R. P. Stanley, Factorization of permutations into n-cycles, Discrete Math. 37(2-3) (1981) 255-262.

[10] W. A. Stein et al., Sage Mathematics Software (Version 4.8), The Sage Development Team, http://www.sagemath.org.

[11] O. Tout, Polynomialité des coefficients de structure des algèbres de doubles-classes. Ph.D thesis, Université de Bordeaux, 2014.

[12] O. Tout, A general framework for the polynomiality property of the structure coefficients of doubleclass algebras, J. Algebr. Comb. 45(4) (2017) 1111-1152. 